\title{
Parámetros hematológicos de pacientes con fibrosis quística de la costa caribe colombiana: análisis y asociación con aislamiento de pseudomonas aeruginosa
}

\section{Zoraya Rodríguez Escobar ${ }^{1}$, Luis Veloza Cabrera², Javier Baena Del Valle ${ }^{3}$, Enrique Ramos ${ }^{4}$, Claudio Gómez Alegría ${ }^{5}$, Doris Gómez Camargo ${ }^{6}$}

\author{
1. Bacterióloga, Especialista en Hematología y Banco de Sangre, Grupo de investigación UNIMOL, Facultad de Medicina, \\ Universidad de Cartagena (Colombia). \\ 2. Médico, Grupo de investigación UNIMOL, Facultad de Medicina, Universidad de Cartagena (Colombia). \\ 3. Médico, Grupo de investigación UNIMOL, Facultad de Medicina, Universidad de Cartagena (Colombia). \\ 4. Médico, Magíster en Salud Pública, Grupo de investigación UNIMOL, Facultad de Medicina, \\ Universidad de Cartagena (Colombia). \\ 5. Bioquímico, Doctor en Bioquímica y Biología Molecular, Profesor asociado e investigador, Departamento de Farmacia, \\ Facultad de Ciencias, Grupo de investigación UNIMOL, Universidad Nacional de Colombia (Colombia). \\ 6. Bacterióloga, Especialista en Educación Médica, Magister en Microbiología Clínica, Doctora en Bioquímica y Biología \\ Molecular. Facultad de Medicina, Universidad de Cartagena (Colombia).
}

Correspondencia: degomez@hotmail.com

Recibido: 02-03-09 / Aceptado: 01-06-09

\begin{abstract}
Resumen
El hemograma es una herramienta fundamental en el control de pacientes con fibrosis quística porque múltiples factores podrían alterar sus parámetros. El objetivo de éste estudio fue analizar y describir los parámetros hematológicos de pacientes con fibrosis quística y determinar su asociación con la infección causada por Pseudomonas aeruginosa y administración de micronutrientes. A 19 pacientes con historia clínica de fibrosis quística se les realizó un hemograma convencional, cultivo de esputo y examen físico. Siete pacientes (36,8\%) presentaron anemia, fue sintomática en un solo caso y leve en los demás. Cuatro pacientes $(21 \%)$ recibían inadecuada administración de micronutrientes. No hubo asociación significativa entre la presencia de anemia y el régimen de administración de micronutrientes con RR 0,63 IC 95\% $(0,|8-3,8|)$. Cuatro (21\%) presentaron leucocitosis, dos (10,5\%) presentaron eosinofilía.

Se aisló Pseudomonas aeruginosa en 8 pacientes (42\%), encontrándose una asociación significativa entre anemia e infección causada por este patógeno, con un RR de 8,25 IC 95\% (I,22-12,06), no siendo significativa la relación entre ésta y los valores de leucocitos. La prevalencia de anemia, probablemente de clase ferropénica, fue mayor que la reportada en la literatura y puede relacionarse quizás con la infección por Pseudomonas aeruginosa, mientras que la administración de micronutrientes posiblemente no se relaciona. La administración crónica de medicamentos puede ser uno de los factores importantes que influyan en los parámetros hematológicos.
\end{abstract}

Palabras clave: anemia, fibrosis quística, leucocitosis, micronutrientes, Pseudomonas aeruginosa. 


\begin{abstract}
Hemogramme is a fundamental tool to asses cystic fibrosis (CF) patients, because multiple factors could change its parameters. The aim of this study was to analyze and describe hematologic patterns of CF patients and determinate their association with infection by Pseudomonas aeruginosa and administration of micronutrients. A conventional Hemogramme, sputum culture, medical history and physical examination were performed to 19 CF patients. 7 patients (36.8\%) presented anemia, only symptomatic in one patient and mild in all cases. 4 patients $(21 \%)$ received an inadequate supplementation with micronutrients. It wasn't a significant association between anemia and micronutrients supplementation regimen with RR $0,63 \mathrm{Cl} 95 \%(0,18-3,8 \mathrm{I}) .4(21 \%)$ presented leucocytosis, in 2 patients (10.5\%) we found eosinophilia. We isolated Pseudomonas aeruginosa in 8 patients (42\%). Finding a significant association between anemia and infection by Pseudomonas aeruginosa with a un RR of 8,25 C195\%( I,22 - 12,06), not being significant between abnormalities in leucocytes values and Pseudomonas aeruginosa. The prevalence of anemia in our study was higher than what is reported in literature and it's probably a ferropenic anemia, that is maybe related to infection by Pseudomonas aeruginosa, while administration of micronutrients is possibly not related with that. Lots of factors in CF patients influence levels of leucocytes without having any clinical manifestation. Chronic administration of drugs could be an important factor that could alter hematologic parameters.
\end{abstract}

Keywords: anemia, cystic fibrosis, leucocytosis, micronutrients, Pseudomonas aeruginosa.

\section{Introducción}

La Fibrosis Quística (FQ) es la enfermedad genética autosómica recesiva más común de la población caucásica, con una incidencia de uno por cada 2.500 recién nacidos vivos (1). Sin embargo, se ha descrito en casi todos los grupos étnicos con grandes variaciones en su incidencia (2-4). En Colombia se estima que uno de cada 16.000 niños puede estar afectado por la enfermedad (5). Es una enfermedad multisistémica, no obstante, la afección del sistema respiratorio frecuentemente asociada a infección por Pseudomonas aeruginosa condiciona el pronóstico de la enfermedad (6,7), causando entre el $90-95 \%$ de las defunciones de los pacientes con esta enfermedad (8).

Durantelas últimas décadas, la edad media de supervivencia ha aumentado de 14 años en 1969 a 35 años en el 2004 en los Estados Unidos (9). Sin embargo, en Latinoamérica los individuos afectados no sobreviven más allá de la adolescencia, con una edad promedio de supervivencia de 12 años (10). El aumento en la expectativa de vida de los pacientes con FQ se ha incrementado principalmente a los avances en los métodos diagnósticos y a la implementación de medidas terapéuticas oportunas, adecuadas y eficaces $(10,11)$.
En el seguimiento y control de los pacientes con FQ la realización del hemograma completo (hemoglobina, hematocrito, conteo de eritrocitos, leucocitos con su fórmula diferencial y plaquetas) como mínimo una vez al año, constituye una herramienta importante para la detección de deficiencias nutricionales y para la identificación de posibles efectos adversos a medicamentos o toxicidad, principalmente durante la participación de los pacientes en los estudios clínicos. Además, la determinación de los valores de leucocitos tanto en su valor absoluto como relativo nos permite conocer la respuesta fisiológica del paciente a la infección o a cualquier otro proceso inflamatorio no infeccioso (12) y de igual forma, ciertas alteraciones en sus valores nos permiten orientar o sospechar patologías concomitantes (10). Por otro lado, el conteo plaquetario y los parámetros eritrocitarios son fundamentales en caso de complicaciones hepáticas y esplénicas (13), por lo que existen muchos factores que podrían alterar los parámetros hematológicos de estos pacientes, unos inherentes a la enfermedad y otros relacionados a ella.

El objetivo de este estudio fue describir e interpretar los resultados del hemograma de un grupo de pacientes 
con FQ de la región Caribe de Colombia mediante la realización de un hemograma convencional y asociar dichos resultados con el aislamiento de Pseudomonas aeruginosa en cultivo de esputo, cuya infección crónica está relacionada con el deterioro de la función pulmonar y la alta mortalidad, además de asociar estos hallazgos con la administración de suplementos de vitaminas liposolubles y minerales que muestran generalmente absorción deficiente en los enfermos con FQ, tales como las vitaminas A, E, hierro y cobre. Estas condiciones podrían relacionarse con alteraciones en los parámetros hematológicos en este grupo de pacientes.

\section{Materiales y métodos}

Pacientes estudiados: un total de 19 pacientes con diagnóstico de $\mathrm{FQ}$, pertenecientes al programa de Atención Integral a Pacientes con esta patología y su Familia de la ciudad de Cartagena, Colombia, fueron incluidos en el estudio. Este programa fue creado por el grupo UNIMOL de la Universidad de Cartagena.

Métodos: previo consentimiento informado se tomó una muestra de sangre venosa de los individuos, que fue enviada al laboratorio de referencia donde se les realizó un hemograma convencional que incluyó hemoglobina, hematocrito, eritrocitos, leucocitos con fórmula diferencial y conteo de plaquetas. Se determinaron niveles de reticulocitos para el cálculo del índice reticulocitario. Los valores de referencia que se utilizaron para la determinación de los parámetros leucocitarios son los establecidos universalmente según la edad. Los parámetros eritrocitarios se compararon con los valores normales establecidos por la OMS según la edad y género (14). También se llevó a cabo un extendido de sangre periférica para el recuento de reticulocitos e identificación de variaciones en el tamaño y forma celulares. En aquellos pacientes con alteraciones en la concentración de hemoglobina se calculó el índice de producción de retículos multiplicando el porcentaje de reticulocitos por el cociente entre hemoglobina del paciente y la hemoglobina esperada según la edad y el género. Este resultado se dividió entre un factor de corrección de acuerdo al valor del hematocrito: 1 para valor de hematocrito de $45 \%, 1,5$ para $35 \%, 2,0$ para $25 \%$ y 2,5 para $15 \%$. Las anormalidades en los parámetros de laboratorio fueron definidos como valores por fuera del rango de referencia para cada parámetro.

A cada paciente se le realizó una historia clínica completa y examen físico que incluía el registro de la concentración, dosis diaria y continuidad de administración de las vitaminas $\mathrm{A}$ y $\mathrm{E}$ y de los bioelementos hierro y cobre en los suplementos nutricionales recibidos. Se realizó el cálculo de la dosis diaria suministrada de cada uno de ellos, considerando un régimen de administración adecuado de estos nutrientes si las dosis eran de 4.000-10.000 UI/día para la vitamina $A, 100-400 \mathrm{UI} /$ día para la vitamina $\mathrm{E}$, 5-10 mg de hiero y de 5-10 mg de cobre. Se consideró inadecuado el régimen de estas vitaminas y minerales si al menos uno de estos parámetros estaba por debajo del límite inferior del rango normal (15).

Se tomó una muestra de esputo de cada paciente para la identificación de Pseudomonas aeruginosa, cada una de estas fueron cultivadas en medios enriquecidos y selectivos, como Cetrimide, MacConkey, EMB y sangre (Merck, Darmstadt, Germany). Se realizó incubación a $37^{\circ} \mathrm{C}$ por 24 horas. Adicionalmente se sembraron placas de Agar Mueller-Hinton (Merck, Darmstadt, Germany) que se incubaron a $42^{\circ} \mathrm{C}$ por 48 horas, confirmando la naturaleza de Pseudomonas aeruginosa. Después del tiempo de incubación convencional se realizó el análisis fenotípico de las colonias y se permitió el crecimiento en Agar F y P (Oxoid, Cambridge, UK). Las evidencias definitivas se obtuvieron por medio del sistema API 20 NE (bioMérieux Vitek, Hazelwood Mo, EU) el cual se utilizó de acuerdo a las instrucciones de manufactura y los resultados se compararon con la base de datos API confirmando la naturaleza del microorganismo. Sólo se correlacionaron con los resultados los hallazgos clínicos y el examen físico de los pacientes que presentaban anormalidades en los parámetros de laboratorio.

Análisis: se realizaron tablas de frecuencia, medidas de tendencia central para las variables cuantitativas continuas y razones de prevalencia $(\mathrm{RR})$ para determinar la asociación entre las distintas variables. Se utilizó como apoyo el software Microsoft Excel 2003 (Redmond WA, EU).

\section{Resultados}

La edad media de los pacientes fue de 11,3 años, la edad mínima fue de 5 años y la máxima fue de 18 años; 10 
pacientes $(52,6 \%)$ eran de género masculino y $9(47,3 \%)$ pertenecían al género femenino. Ningún paciente presentó síntomas o signos clínicos compatibles con exacerbación pulmonar al momento del examen físico, como incremento en la frecuencia o intensidad de la tos, aumento en la cantidad del esputo o cambios en sus características, aparición o incremento de disnea, fiebre, incremento de la frecuencia respiratoria basal, cambios en la auscultación pulmonar habitual, disminución del apetito o pérdida de peso. Los criterios radiológicos, espirométricos y analíticos de exacerbación pulmonar no fueron identificados en éste estudio. Los 11 parámetros hematológicos determinados en los 19 pacientes con FQ se muestran en la Tabla 1. Los valores fueron comparados con los rangos de referencia universales y el porcentaje de valores anormales de cada parámetro se presentan en la Tabla 2.

En el momento de realización del estudio, 15 de los 19 pacientes $(78,9 \%)$ presentaron al menos uno de los 11 parámetros fuera del rango normal. El parámetro menos alterado fue el de los eosinófilos, que se observó en 2 individuos (10,5\%); el más alterado fue el conteo de monocitos, que fue observado en 14 pacientes $(73,3 \%)$; ningún individuo presentó valores de plaquetas, ni basófilos por fuera de los rangos normales. 4 sujetos (21\%) presentaron un conteo de glóbulos blancos por encima del valor normal, 7 individuos (36,8\%) tuvieron niveles de hemoglobina y hematocrito diagnósticos para anemia. Un sujeto presentó eosinofilia moderada $(574 \mathrm{c} / \mathrm{mL})$ y otro presentó eosinofilia severa $(3960 \mathrm{c} / \mathrm{mL})$. Los 4 sujetos que presentaron linfocitosis fueron de tipo relativa (media de linfocitos: $56,7 \%$ ).

En ocho pacientes $(42,1 \%)$ el índice reticulocitario se encontró elevado sobre 3, de los cuales un $15,8 \%$ no tuvieron anemia. Del 36,8\% de los pacientes con anemia el 26,3\% (5/19) tuvieron un índice reticulocitario elevado mientras que el 10,5\% (2/19) poseía un índice reticulocitario normal. A los 7 pacientes que tuvieron anemia se les realizó anamnesis y examen físico en búsqueda de manifestaciones clínicas de la anemia. En éste estudio sólo un sujeto (5\%) presentó anemia con manifestaciones clínicas leves. Este individuo fue una niña de 8 años de edad, con un nivel de hemoglobina de $10 \mathrm{gr} /$ $\mathrm{dL}$ y un hematocrito de $30 \%$, al momento del estudio presentaba astenia progresiva y palidez mucocutánea.

Tabla1. Parámetros de los 19 pacientes con FQ.

\begin{tabular}{cccccccccccccc} 
Pte & Edad & Sexo & Hb & Hto & GR & Ret & GB & Lin & Neu & Eos & Bas & Mon & Plq \\
\hline 1 & 14 & M & 11.0 & 33 & 3.6 & 2,8 & 7.00 & 43 & 60 & 4 & 1 & 4 & 357 \\
2 & 13 & M & 13,6 & 41 & 4.4 & 0,8 & 8.20 & 64 & 27 & 7 & 0 & 2 & 315 \\
3 & 16 & M & 13,6 & 41 & 4.4 & 1,6 & 5.15 & 59 & 37 & 3 & 0 & 1 & 252 \\
4 & 5 & F & 12,3 & 37 & 4.1 & 2,8 & 10.40 & 34 & 56 & 2 & 0 & 6 & 231 \\
5 & 11 & M & 10.0 & 30 & 3.3 & 2,2 & 11.00 & 45 & 13 & 36 & 0 & 4 & 315 \\
6 & 18 & F & 11.0 & 33 & 3.6 & 1,8 & 8.60 & 31 & 64 & 2 & 1 & 1 & 546 \\
7 & 14 & M & 11.0 & 33 & 3.6 & 2.0 & 10.50 & 43 & 54 & 0 & 0 & 3 & 336 \\
8 & 5 & F & 12,3 & 37 & 4.0 & 1.0 & 8.15 & 53 & 40 & 5 & 1 & 1 & 357 \\
9 & 14 & F & 14,3 & 43 & 4.6 & 2,2 & 14.25 & 18 & 72 & 2 & 0 & 7 & 357 \\
10 & 11 & F & 14,3 & 43 & 4.6 & 1.0 & 19.45 & 15 & 78 & 2 & 1 & 3 & 294 \\
11 & 8 & F & 10,8 & 33 & 3.6 & 2,6 & 10.50 & 29 & 64 & 2 & 0 & 2 & 294 \\
12 & 11 & M & 13,3 & 40 & 4.1 & 2,6 & 7.60 & 32 & 56 & 4 & 1 & 3 & 300 \\
13 & 13 & M & 14.0 & 40 & 4.3 & 1,4 & 8.75 & 51 & 43 & 1 & 0 & 5 & 252 \\
14 & 15 & M & 13,6 & 41 & 4.4 & 1,6 & 9.25 & 27 & 68 & 2 & 2 & 1 & 441 \\
15 & 8 & F & 10.0 & 30 & 3.3 & 2,6 & 7.00 & 25 & 58 & 3 & 0 & 1 & 390 \\
16 & 8 & F & 10.0 & 30 & 3.3 & 2,2 & 10.60 & 33 & 62 & 3 & 0 & 2 & 231 \\
17 & 12 & M & 12.0 & 36 & 3.9 & 2.0 & 12.00 & 28 & 60 & 4 & 1 & 3 & 289 \\
18 & 12 & F & 13,6 & 41 & 4.4 & 1,2 & 5.00 & 28 & 55 & 3 & 2 & 2 & 160 \\
19 & 7 & M & 13.0 & 36 & 4.2 & 1,2 & 7.00 & 25 & 56 & 4 & 1 & 2 & 340 \\
\hline
\end{tabular}

Hb: hemoglobina (g/dl); Hto: hematocrito (\%); GR: glóbulos rojos (106 cél/mm3); Ret: reticulocitos (\%); GB: glóbulos blancos (103 cel/mm3); Lin: linfocitos (\%); Neu: neutrófilos (\%); Eos: eosinófilos (\%); Bas: basófilos (\%); Mon: monocitos (\%); Plq: plaquetas (103 cél/mm3). 
Tabla 2. Proporción de pacientes con parámetros hematológicos alterados.

\begin{tabular}{|c|c|c|c|c|c|c|}
\hline \multirow[t]{2}{*}{ Parámetro } & \multicolumn{2}{|c|}{ Bajo límite inferior } & \multicolumn{2}{|c|}{ Sobre límite superior } & \multicolumn{2}{|c|}{ Total } \\
\hline & No. & $\%$ & No. & $\%$ & No. & $\%$ \\
\hline G. blancos (cél. $/ \mathrm{mm}^{3}$ ) & 0 & 0 & 4 & 21 & 4 & 21 \\
\hline G. rojos (cél./mm³) & 7 & 36,8 & 0 & 0 & 7 & 36,8 \\
\hline Plaquetas (cél./mm³) & 0 & 0 & 4 & 21 & 4 & 21, \\
\hline Linfocitos (\%) & 0 & 0 & 0 & 0 & 0 & 0 \\
\hline Neutrófilos (\%) & 1 & 5,3 & 2 & 10,5 & 3 & 15,8 \\
\hline Basófilos (\%) & 0 & 0 & 0 & 0 & 0 & 0 \\
\hline Eosinófilos (\%) & 0 & 0 & 2 & 10,5 & 3 & 10,5 \\
\hline Monocitos (\%) & 0 & 0 & 0 & 0 & 0 & 0 \\
\hline Hemoglobina (g/dl) & 7 & 36,8 & 0 & 0 & 7 & 36,8 \\
\hline Hematocrito (\%) & 7 & 36,8 & 0 & 0 & 7 & 36,8 \\
\hline
\end{tabular}

Tabla 3. Distribución de los casos de anemia según edad y sexo.

\begin{tabular}{ccccccc} 
Edad (años) & \multicolumn{2}{c}{ Sexo masculino } & \multicolumn{2}{c}{ Sexo femenino } & \multicolumn{2}{c}{ Total } \\
& No. & $\%$ & No. & $\%$ & No. & $\%$ \\
\hline$<6$ & 0 & 0 & 0 & 0 & 0 & 0 \\
$\mathbf{6}-\mathbf{1 4}$ & 3 & 15.8 & 3 & 15.8 & 6 & 31.6 \\
$>14$ & 1 & 5.3 & 0 & 0 & 1 & 5.3 \\
Total & 4 & 21.0 & 3 & 15.8 & 7 & 36.8 \\
\hline
\end{tabular}

Por otro lado, 6 de los 7 sujetos con anemia $(31,6 \%)$ negaron antecedentes de hemorragias digestivas, ya sea melenas, hematemesis o sangre oculta en heces, del mismo modo sangrado anormal en cualquier otra localización. Uno de los 7 individuos con anemia tenía hallazgos de cirrosis hepática con hipertensión portal, esplenomegalia y várices esofágicas, sin embargo no presentó sangrado macroscópico y no existen datos sobre la realización de examen de sangre oculta en heces durante la realización de este estudio. Este individuo de sexo masculino, de 14 años, sin síntomas ni signos de anemia tuvo una hemoglobina de $11 \mathrm{gr} / \mathrm{dL}$, hematocrito de $33 \%$ e índice reticulocitario sobre 3. No presentó ningún otro síntoma o signo importante al examen físico.

De los 7 pacientes $(36,8 \%)$ con anemia, 4 fueron de género femenino y la edad media fue de 11,5 años (edad mínima 8 años y edad máxima 18 años). La distribución de los casos de anemia según edad y género se presentan en la Tabla 3. La anemia fue leve en todos los casos en relación con los niveles de hemoglobina para la edad $(\mathrm{Hb}$ 10-12gr/dL para niños de 6-14 años, Hb 11-13gr/dL para hombres mayores de 14 años y $\mathrm{Hb} 10-12 \mathrm{gr} / \mathrm{dL}$ para mujeres mayores de 14 años. Se detectó una prevalencia de Pseudomonas aeruginosa en esputo en un $42 \%$, con una edad media de 11,6 años y una distribución de acuerdo a edad y género que se muestra en la Tabla 4.

Cuatro pacientes de los 19 (21\%) recibían un inadecuado régimen de administración de las vitaminas y minerales considerados en este estudio, mientras que 15 individuos (79\%) recibían un régimen adecuado de suplementos de estas vitaminas y minerales.

La asociación entre administración de suplementos de vitaminas y minerales de manera inadecuada y la presencia de anemia no fue significativa RR 0,63 (IC: 0,18-3,81). Se encontró una asociación positiva entre el aislamiento de Pseudomonas aeruginosa y la presencia de anemia, con un RR de 8,25 IC 95\% (1,22-12,06), p=0,003, la asociación causal fue significativa. Se halló una asociación 
entre leucocitosis y cultivo de Pseudomonas aeruginosa positivo no significativa, con un RR de 1,375 IC $95 \%$ (0,81-12,06), Tabla 5.

El individuo que presentó la eosinofilia severa $(3960 \mathrm{cc} /$ $\mathrm{mL})$ también tuvo anemia leve $(\mathrm{Hb}: 10 \mathrm{gr} / \mathrm{dL})$ y cultivo de Pseudomonas aeruginosa positivo en esputo. El sujeto que presentó la eosinofilia moderada $(574 \mathrm{cc} / \mathrm{mL})$ tuvo además linfocitosis relativa (64\%), pero no tenia anemia ni cultivo de Pseudomonas aeruginosa positivo en esputo. De los 2 casos de neutrofilía, uno presentó leucocitosis $(19.450 \mathrm{cc} / \mathrm{ml})$, cultivo de Pseudomonas aeruginosa positivo en esputo y anemia. El otro caso tuvo cultivo de Pseudomonas aeruginosa negativo en esputo, pero no presentó anemia.

\section{Discusión}

Este es el primer estudio colombiano donde los parámetros hematológicos y su comparación en pacientes diagnosticados con FQ y con la población en general se realiza en la ciudad de Cartagena, Colombia. Pueden existir factores de tipo ambiental, nutricional o socioeconómico, cuya interacción influyen tanto en la alteración del cuadro clínico y en la expectativa de vida de los pacientes. Es importante considerar que en la mayoría de los estudios internacionales realizados en pacientes con FQ comparan los parámetros hematológicos con los valores de referencia usados para la población general sin FQ. Se desconoce si los valores de referencia hematológicos necesitan ser enfocados para considerar la fisiología y fisiopatología de los pacientes con esta enfermedad.

Existen muy pocos estudios con relación a la prevalencia de anemia en la población con FQ (16). Uno de ellos, el realizado en Lima, Perú, con 42 pacientes, entre los años 1991 al 2001, se reportó una prevalencia de anemia de $38,5 \%$ (17), cifra comparable a la encontrada en esta investigación, que fue del 36,8\%. Esto puede indicar que existen factores y/o mecanismos etiopatológicos o fisiopatológicos similares y múltiples factores de riesgo que son capaces de producir anemia como la malabsorción de vitaminas y minerales, las infecciones crónicas, los trastornos inflamatorios no infecciosos y los efectos adversos a medicamentos, entre otros (18).

Tabla 4. Distribución de los casos de positivos de Pseudomonas aeruginosa en esputo según edad y sexo.

\begin{tabular}{ccccccc} 
Edad (años) & \multicolumn{2}{c}{ Sexo masculino } & \multicolumn{2}{c}{ Sexo femenino } & \multicolumn{2}{c}{ Total } \\
& No. & $\%$ & No. & $\%$ & No. & $\%$ \\
\hline Menor de 6 años & 0 & 0 & 0 & 0 & 0 & 0 \\
$6-10$ años & 3 & 15,8 & 0 & 0 & 3 & 15,8 \\
$11-17$ años & 1 & 5,3 & 3 & 15,8 & 4 & 21 \\
Mayor de 17 años & 1 & 5,3 & 0 & 0 & 1 & 5,3 \\
Total & 5 & 26,2 & 3 & 15,8 & 8 & 42 \\
\hline
\end{tabular}

Tabla 5. Asociación entre anemia, régimen de suplementos inadecuado y cultivo de $P$. aeruginosa y asociación entre leucocitosis y cultivo de $P$. aeruginosa positivo.

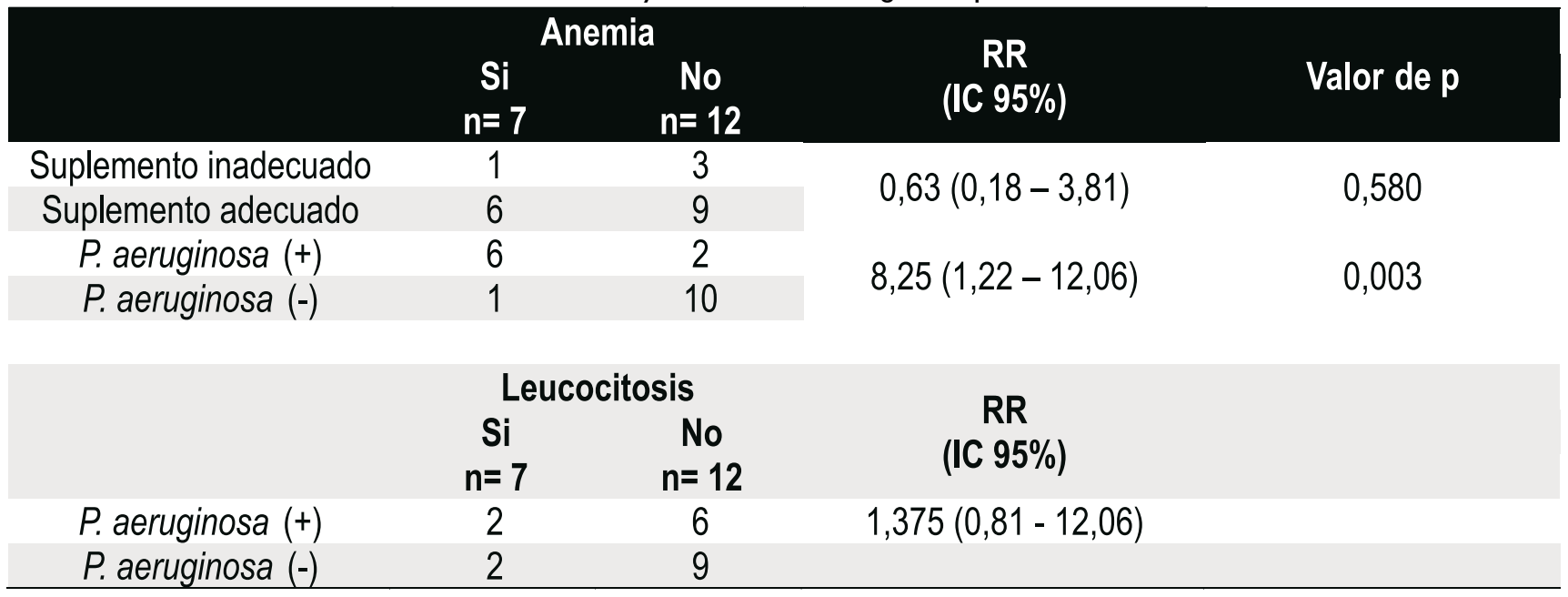


No se encontró una diferencia significativa en los casos de anemia según el género, probablemente porque ningún individuo sobrepasó los 18 años de edad. Sólo se ha reportado esta alteración en adultos, especialmente en mujeres con $\mathrm{FQ}$, ya que en la población general el 37\% presentan anemia debido principalmente a las pérdidas de hierro causadas por los períodos menstruales, mientras que en los hombres adultos sólo se presenta en un $18 \%$ (19). A pesar de esto no se sabe si la interacción entre los factores inherentes al género y los relacionados con la enfermedad influirían en éstas tasas.

La anemia, en relación con los valores de hemoglobina relacionados con la edad, fue leve en todos los casos reportados. La presencia de anemia severa es rara en los pacientes con FQy se ha reportado una frecuencia de ésta clínicamente significativa en un $4 \%$ de los niños con la enfermedad (16). En este estudio se encontró un caso de anemia sintomática (5\%). Se ha reportado un caso de un recién nacido de 2,5 meses con FQ cuya principal y primera manifestación fue una anemia macrocítica con hemoglobina de $6.5 \mathrm{gr} / \mathrm{dL}$ asociada a desnutrición e hipoproteinemia, pero con mínimos síntomas pulmonares. A pesar de un tratamiento adecuado, el paciente falleció por una enfermedad hepática y pulmonar severa a los 6 años de edad (20). La anemia temprana podría deberse a una deficiencia de vitamina $\mathrm{E}$ que provocaría una anemia tipo hemolítica o a una deficiencia de ácido fólico ya que es macrocítica. Más investigaciones son necesarias para clarificar el mecanismo de la anemia temprana en personas con FQ y evaluar el valor pronóstico de una anemia clínicamente significativa en los lactantes con FQ.

En el 85\% de los individuos con FQ se puede llegar a desarrollar una disfunción pancreática exocrina debido a la alteración en la secreción de cloruro dentro de los ductos pancreáticos que conlleva a obstrucción y autodestrucción del tejido pancreático $(21,22)$. Esta alteración de la función pancreática exocrina, sumada a la disfunción intestinal y hepática, produce una malabsorción de nutrientes principalmente de vitaminas liposolubles (A, $\mathrm{D}, \mathrm{E}, \mathrm{K})$ y minerales como calcio, cobre, zinc, hierro y selenio (23). De éstas, la deficiencias de vitaminas A y E y de bioelementos como hierro y cobre pueden causar anemia (15). De igual forma, si las dosis diarias no son las adecuadas, los pacientes con FQ pueden sufrir de deficiencias de vitaminas hidrosolubles causantes de anemia (B12, Acido fólico).

La asociación entre administración de suplementos como vitaminas A y E, y de bioelementos como el hierro y el cobre de manera inadecuada y la presencia de anemia no fue significativa RR 0,63 (IC: 0,18-3,81). Los resultados de esta asociación no son concluyentes para catalogar la administración de suplementos de estos micronutrientes como un factor de riesgo o protector, además las manifestaciones clínicas por deficiencias vitamínicas son raras en pacientes con FQ (15). Probablemente existan otros factores ajenos a la administración de estos suplementos que pueden relacionarse con la presencia de anemia. Es importante anotar que en esta investigación no se tuvo en cuenta las dosis diarias de micronutrientes aportados por los alimentos y la presencia de insuficiencia pancreática que varía entre los distintos pacientes, ni se valoró las concentraciones de las vitaminas liposolubles (A,D,E, K). Consideramos importante controlar las dosis diarias de estas vitaminas para mantener unos niveles séricos adecuados, así como; la deficiencia de hierro y demás minerales, pues un adecuado régimen nutricional puede inhibir el deterioro de la función pulmonar, mejorando la calidad de vida y expectativa de supervivencia de los pacientes (26).

La anemia causada por deficiencia de cobre, también se acompaña de neutropenia, alteraciones cardiacas, debilidad muscular, atrofia pancreática, alteraciones inmunes y en el tejido conectivo (15). Interesantemente un sólo paciente presentó neutropenia $(1430 \mathrm{cc} / \mathrm{mL})$ y anemia leve (Hb: $10 \mathrm{gr} / \mathrm{dL}$ ) en este estudio, por lo cual, aunque no existan los signos clínicos de la deficiencia de cobre, cabe la posibilidad de realizar los exámenes paraclínicos pertinentes para confirmar o descartar esta deficiencia.

Aunque el hecho de que se haya encontrado en 5 sujetos de los 7 con anemia un índice reticulocitario elevado sugiere que la anemia prevalente es de tipo regenerativa, como las causadas por hemorragias o anemias hemolíticas (27), no se podría determinar cuál de estos tipos está presente sin otros exámenes complementarios que no se realizaron en éste estudio por ausencia de recursos necesarios.

En la población general, de todas las anemias, el 50\% se deben a déficit de hierro, y en los pacientes con FQ es más frecuente, aunque su significado clínico es incierto. 
La prevalencia de deficiencia de hierro se incrementa aproximadamente de un 33\% de los casos pediátricos a un $60 \%$ en la población adulta, y normalmente es atribuida a factores gastrointestinales, a la pobre ingesta en la dieta y la combinación con inflamación crónica (28).

Aunque la prevalencia de deficiencia de hierro sea alta en la población con FQ no es posible relacionarla directamente con la presencia de anemia en este estudio ya que para establecer si los casos de anemia encontrados en esta investigación son por déficit de hierro, se tendrían que determinar los parámetros de la cinética del hierro (ferritina, transferrina, capacidad de transferrina, porcentaje de saturación de transferrina) que confirmarían el estado de ferropenia. De igual forma los índices eritrocitarios ayudarían a una aproximación diagnóstica de la anemia en éste caso, igualmente en los pacientes con FQ las infecciones crónicas y trastornos inflamatorios podrían estar asociadas a la presencia de anemia. Citoquinas como el factor de necrosis tumoral, IL- 1, IL - 6 e interferones se han involucrado en el desarrollo de la anemia de enfermedades crónicas al disminuir la vida media del eritrocito, alterar la respuesta a la eritropoyetina y la movilización de hierro desde las reservas reticuloendoteliales (29).

Además, hay que tener en cuenta que los pacientes con FQ reciben crónicamente muchos medicamentos y antibióticos como cloranfenicol y trimetoprim / sulfametosaxol que también podrían causar disminución de los niveles de hemoglobina y de hematocrito (30). En este estudio no se documentaron los esquemas de tratamientos que recibieron los pacientes, pero sirve de base para la investigación de posibles efectos adversos a estos medicamentos.

Lo anterior permite anotar que para realizar una aproximación al diagnóstico diferencial de la anemia y sospechar de una deficiencia nutricional en particular se necesitarían los índices eritrocitarios como el volumen corpuscular medio (VCM), hemoglobina corpuscular media (HCM), concentración corpuscular media de hemoglobina (CCMH) y ancho de distribución eritrocitaria (ADE) cuyas variaciones nos guiarían hacia un tipo de anemia en particular, ya que no existe evidencia clínica de ningún déficit. En este estudio no fue posible determinar estos índices por ausencia del equipo necesario en nuestra institución.
De otro lado, la enfermedad pulmonar crónica constituye el $95 \%$ de los casos de mortalidad en los pacientes con FQ y se caracteriza por la colonización de la vía aérea por la bacteria oportunista Pseudomonas aeruginosa a temprana edad (31) y el desarrollo de infección crónica está relacionado con la habilidad del microorganismo para proliferar a pesar de la respuesta inmune del huésped y de esquemas de tratamientos agresivos (32).

Se ha identificado una relación indirecta entre la severidad de la enfermedad pulmonar y el grado de desnutrición y deficiencia de hierro en pacientes con FQ. Sin embargo, es posible que la mala absorción de los antioxidantes de la dieta y la deficiencia de hierro sistémica pueda contribuir a la destrucción pulmonar por alteración de los sistemas de defensa inmune y antioxidantes dentro de la vía aérea del paciente con FQ (28).

Pseudomonas aeruginosa se encuentra en el 60\% de los pacientes del registro americano, pero las cifras oscilan desde 69\% en Irlanda y 62\% en Francia, hasta 44\% en Nueva Zelanda (33) y 48\% Canadá (34). En este trabajo, se detectó una prevalencia de aislamiento de Pseudomonas aeruginosa en esputo de $42 \%$. Estas prevalencias están influenciadas principalmente por la edad de los sujetos estudiados, ya que este factor influye en la colonización por los distintos gérmenes. Pseudomonas aeruginosa se aisla en el 30\% de los niños de 2- 5 años, en el $40 \%$ de los niños de 6-10 años, en el 60\% entre los 11 a 17 años y la cifra asciende hasta un 70-80\% entre los 18-24 ańos (11). No obstante, en el estudio realizado en las unidades de la Comunidad de Madrid, el 33,1\% (126 casos) del total de pacientes presentaron colonización por Pseudomonas aeruginosa; en los menores de 18 ańos fue el 24,4\% observándose claramente la influencia de la edad en la colonización (11), mientras que en nuestro estudio, en que todos los individuos eran menores de 18 ańos, la prevalencia fue de $42 \%$ como se mencionó anteriormente. Estas prevalencias tan disímiles se podrían deber en parte al manejo integral del paciente y a la política agresiva antibiótica frente a esta bacteria practicada en las unidades europeas de FQ desde hace varios años.

En este estudio la edad media de los casos de aislamiento de Pseudomonas aeruginosa en esputo fue de 11,6 años y una distribución de acuerdo a edad: menor de 5 años: ningún 
paciente, 6-10 años: 3 pacientes, entre los $11-17$ años: 4 pacientes y 1 paciente mayor de 17 años. Los resultados también reflejan una proporción ascendente de los casos según la edad como reporta la literatura, considerando que un único individuo sobrepasaba los 17 años.

En relación con la distribución de acuerdo al género $5 / 8$ son mujeres $(62,5 \%)$ y $3 / 8$ son hombres $(37,5 \%)$. Los 3 pacientes de 6-10 años son de género femenino, lo que confirma lo reportado en la literatura que las niñas son colonizadas a edades más tempranas en relación con los individuos de género masculino, por razones aún no esclarecidas (11).

La asociación entre presencia de anemia y cultivo de Pseudomonas aeruginosa positivo fue significativo RR $8,25$ (IC: $1,22-12,06)(\mathrm{p}=0,003)$. No se ha establecido un efecto directo causal de infección por Pseudomonas aeruginosa y déficit de hierro. Sin embargo, la habilidad de la bacteria para obtener el hierro extracelular de los tejidos del huésped para su crecimiento y desarrollo de virulencia por la secreción de gránulos unidores de hierro llamados sideróforos sugieren que éste organismo podría jugar un papel directo en la depleción de las reservas de hierro (28). Si la pérdida de hierro dentro de la vía aérea ocurre como una consecuencia de la infección por Pseudomonas aeruginosa, entonces el volumen del esputo expectorado diariamente y su alto contenido de hierro podría contribuir de manera importante a la deficiencia de hierro en los pacientes con $F Q$, ya que se sabe que el esputo y el fluido de líquido broncoalveolar de individuos con FQ contienen cantidades aumentadas de hierro. Existiendo un círculo vicioso entre colonización por Pseudomonas aeruginosa, depleción del hierro y anemia.

Las causas de leucocitosis en la población con FQ pueden ser las mismas que en la población general (35). Las infecciones de la vía respiratoria serían las causas más frecuente junto con las alteraciones en el trasporte mucociliar y la consiguiente infección bacteriana. En éste estudio 4 pacientes (21\%) tuvieron leucocitosis probablemente como respuesta a una infección crónica de la vía respiratoria, ya que no hubo signos ni síntomas clínicos de exacerbación infecciosa ni en otra localización. Además, hay que considerar que medicamentos como los corticoides pueden elevar los niveles de leucocitos en estos pacientes.
La asociación entre la presencia de leucocitosis y de Pseudomonas aeruginosa no fue significativa RR 1,375 (IC: 0,81-12,06), en parte porque los enfermos con FQ tienden a ser colonizados por otras bacterias como: Staphilococcus aureus, Haemophilus influenzae, el complejo Burkholderia cepacia, etc. que también pueden provocar una respuesta inmunológica con leucocitosis (36). Aunque no hay evidencia de inmunodeficiencia en los pacientes con FQ, si existen alteraciones del sistema inmune contra Pseudomonas aeruginosa: en primer lugar se presenta una respuesta significativa humoral dirigida frente a los antígenos de este patógeno, no pudiendo eliminarse de la vía aérea y los anticuerpos dirigidos contra esta bacteria podrían ser el primer marcador de infección en los individuos afectados (37). Sin embargo, mecanismos como la opsonización y la fagocitosis bacteriana estaría disminuida.

En un paciente la neutrofília $(15.161 \mathrm{cc} / \mathrm{mL})$, la leucocitosis $(19.450 \mathrm{cc} / \mathrm{mL}$ ) y el cultivo de Pseudomonas aeruginosa positivo en esputo podría indicar que la infección por este germen es la causante de estas anormalidades hematológicas. De igual forma, la coinfección con otra bacteria puede causar este aumento en el número de leucocitos y neutrófilos. Cuatro pacientes presentaron linfocitosis relativa, la cual, igual que en la población sin FQ puede ser debida en parte a infecciones virales respiratorias que no fueron clínicamente evidentes al momento de la anamnesis y al examen físico.

Existen pocos estudios que aclaren el potencial significado de los virus respiratorios. Algunos estudios sugieren que alrededor de un $40 \%$ de las exacerbaciones en la FQ estarían asociadas a algún virus respiratorio (virus respiratorio sincitial e influenza) (38). Estos virus conducirían al deterioro de la función pulmonar y a la progresión de la enfermedad predisponiendo secundariamente a la infección y colonización bacteriana, ya que los virus causarían un daño en el epitelio respiratorio que aumentaría su la adherencia de bacterias como S. aureus, H. influenzae, S. pneumoniae y P. aeruginosa (37).

Dos pacientes de los 19 presentaron eosinofilia, uno moderada $(574 \mathrm{cc} / \mathrm{mL})$ y otro severa $(3960 \mathrm{cc} / \mathrm{mL})$. Aumentos en los niveles de eosinófilos se deben con mayor frecuencia a infecciones parasitarias y enfermedades 
alérgicas, sin embargo, no hubo evidencia en la anamnesis ni en la exploración física de éstas condiciones. Pese a esto, fármacos como las penicilinas, aminoglucósidos, la clindamicina y cefalosporinas, comúnmente usados en los pacientes con FQ, pueden también causar eosinofilia (30). Unos niveles altos de estas células en pacientes con FQ acompañados de otros criterios como: sibilancias, fiebre, malestar, secreciones bronquiales espesas y oscuras, hemoptisis, infiltrados broncopulmonares $>1 \mathrm{~cm}$ de diámetro y colapso segmentario, Ig E elevada, Aspergillus fumigatus cultivado en secreciones son diagnósticos de Aspergilosis broncopulmonar (ABP) (30). Los pacientes que tuvieron eosinofilia no presentaron los criterios clínicos de ABP, siendo necesario la realización de los exámenes paraclínicos para descartar totalmente esta entidad. Además, la cirrosis hepática puede causar aumento en las cifras de eosinófilos, pero ninguno de los individuos con eosinofilia mostró evidencia en la anamnesis ni en la exploración física de esta condición y el sujeto que tuvo cirrosis no presentó eosinofilia.

Un solo paciente presentó neutropenia $(1430 \mathrm{cc} /$ $\mathrm{ml}$ ), que probablemente podría deberse al consumo de múltiples medicamentos como vancomicina, trimetoprim/ sulfametosaxol y cefaclor, entre otros (30). A este respecto cabría investigar los esquemas de tratamientos recibidos por este sujeto para determinar si existe o no asociación con la presencia de neutropenia.

Para finalizar, el nivel plaquetas no se encontró alterado en ningún individuo, lo que se esperaba según la anamnesis y la exploración física de 18 de los 19 individuos, principalmente en relación con la afección hepática y esplénica. Un solo individuo tuvo cirrosis hepática con hipertensión portal y várices esofágicas, sin embargo, no presentó trombocitopenia, pero sí una anemia leve, que en este caso puede deberse a hemorragia digestiva leve, no evidente macroscópicamente o ser un signo de hiperesplenismo. En éste caso la realización de un hemograma es fundamental para buscar los signos de hiperesplenismo, como anemia y trombocitopenia lo que es crucial al momento la instauración de medias terapéuticas durante complicaciones como hemorragias digestivas por várices que es la complicación más frecuente de la cirrosis (13).

\section{Agradecimientos}

A todos los pacientes y familiares que hacen parte del Programa de Atención Integral a Pacientes con Fibrosis Quística del grupo UNIMOL por haber participado en el estudio, al Dr. Fabio Anaya, a la Dra. Dacia Malambo y Melissa Arrieta Franco por su asistencia en este trabajo y la Facultad de Medicina de la Universidad de Cartagena.

\section{Referencias}

1. Ratjen F, Döring G. Cystic fibrosis. Lancet. 2003;361:681-689.

2. Bobadilla J, Macek M, Fine J, Farrell P. Cystic fibrosis: a worldwide analysis of CFTR mutations: correlation with incidence data and application to screening. Hum Mutat. 2002;19:575-606.

3. Keyeux G, Rodas MC, Bienvenu T, Garavito P, Vidaud, Sanchez $\mathrm{D}$, et al. CFTR mutations in patients from Colombia: Implications for local and regional molecular diagnosis programs. Hum Mutat. 2003;22:259.

4. Organización Mundial de la Salud (OMS). The molecular genetic epidemiology of cystic fibrosis. Ginebra: WHO; 2004.

5. Instituto de Genética, Universidad Nacional de Colombia. Fibrosis quística, Fibrosis quística en Colombia. Disponible en: http://www.genetica. unal.edu.co/pages/fibrosis.html. Consultado: 11 de Julio 2008.

6. Heijerman H. Infection and inflammation in cystic fibrosis: A short review. J Cyst Fibros. 2005;4:3-5.

7. Taccetti G, Campana S, Festini F, Mascherini M, Doring G. Early eradication therapy against Pseudomonas aeruginosa in cystic fibrosis patients. Eur Respir J. 2005;26:458-461.

8. Jiménez TS, Bousoño GC, Ruiz DP. Protocolos de Patología respiratoria. Patología pulmonar en la fibrosis quística. Bol Soc Catalana Pediatr. 2007;47 Suppl 2:S7-13.

9. Cystic Fibrosis Foundation Patient Registry, 2000. Annual Data Report. Bethesda, Maryland; 2001.

10. Sánchez I, Perez M, Boza M, Lezana V, Vila M, Repetto G, et al. Consenso nacional de fibrosis quística. Rev Chil Pediatr. 2001;72:356-380.

11. Girón R, Salcedo A, Casanova A. Antecedentes históricos y epidemiología. En: Girón R, Salcedo A, eds. Monografía de Fibrosis Quística. Madrid: Ergón; 2005. p. 9-20.

12. Becker A. Interpretación del hemograma. Rev Chil Pediatr. 2001;72:460-465.

13. García M, Garfia C, Manzanares J. Fibrosis quística. Monografías Neumomadrid. $1^{\text {a }}$ ed. Madrid: Ergón; 2005.

14. Organización Mundial de la Salud (OMS). Iron deficiency anaemia: assessment, prevention, and control. A guide for program managers. Guía Tecnica. Ginebra: OMS; 2004. Xiii.

15. Sinaasappel M, Stern M, Littlewood J, Wolfe S, Steinkamp G, Heijerman $\mathrm{H}$, et al. Nutrition in patients with cystic fibrosis: a European consensus. J Cystic Fibrosis. 2002;1:51-75.

16. Wilfond B, Farrell P, Laxova A. Severe haemolytic anemia associated with vitamine E deficiency in infants with cystic fibrosis. Clin Pediatr. 1994;33:2-7.

17. Torres D. Estudio clínico epidemiológico de la fibrosis quística en el Instituto de Salud del Niño, Lima 1991 - 2002. Paediatrica. 2002;4:-15.

18. Dowsett J, Tully O. Cystic fibrosis. En: Caballero B, Allen L, Prentice A, editores. Encyclopedia of Human Nutrition. $2^{\circ}$ ed. Boston: Elsevier/Academic Press; 2005. p 494-501.

19. Stoltzfus RJ. Iron-deficiency anemia: reexamining the nature and magnitude of the public health problem. Summary: implications for research and programs. J Nutr. 2001;131(2 Suppl 2): 697S-700S.

20. Kahre T, Teder M, Panov M, Metspalu A. Severe CF manifestation with anaemia and failure to thrive in a 394delTT homozygous patient. J Cyst Fibros. 2004;3:58-60. 
21. Steven M. Rowe M, Miller S. Mechanisms of disease cystic fibrosis. N Engl J Med. 2001;352:1992-2001.

22. Ramsey B, Accurso F, Cutting G, Welsh M. Cystic fibrosis. In: Valle D, Beaudet A, Vogelstein B, Kenneth K, Antonarakis S, Ballabio A, editors. The metabolic and molecular bases of inherited disease. $1^{\text {a }}$ ed. Baltimore: Mc Graw Hill; 2007. p. 121- 88.

23. Dodge JA, Turck D. Cystic fibrosis: nutritional consequences and management. Best Pract Res Clin Gastroenterol. 2006;20:531-546.

24. Lancellotti L, D’Orazio C, Mastella G, Mazzi G, Lippi U. Deficiency of vitamins $\mathrm{E}$ and $\mathrm{A}$ in cystic fibrosis is independent of pancreatic function and current enzyme and vitamin supplementation. Eur $\mathrm{J}$ Pediatr. 1996;155:281-285.

25. Swann IL, Kendra JR. Anaemia, vitamin E deficiency and failure to thrive in an infant. Clin Lab Haematol. 1998;20:61-63.

26. Winklhofer-Roob BM. Cystic fibrosis: nutritional status and micronutrients. Curr Opin Clin Nutr Metab Care. 2000;3:293-297.

27. Rodríguez J, Hernández R, López R. Aproximación al diagnostico de las anemias. BSCP Can Ped. 2001;25:251-255.

28. Reid DW, Withers NJ, Francis L, Wilson JW, Kotsimbos TC. Iron deficiency in cystic fibrosis: relationship to lung disease severity and chronic Pseudomonas aeruginosa infection. Chest. 2002;121:48-54.

29. O'connor TM, McGrath DS, Short C, O'donnell MJ, Sheehy M, Bredin CP. Subclinical anaemia of chronic disease in adult patients with cystic fibrosis. J Cyst Fibros. 2002;1:31-34.
30. Segal E, Grenoville M, Macri C, Fernández A. Consenso de fibrosis quística. Arch argent Pediatr. 1999;97:188-219.

31. Davies JC. Pseudomonas aeruginosa in cystic fibrosis: pathogenesis and persistence. Paediatr Respir Rev. 2002;3:128-134.

32. Gómez MI, Prince A. Opportunistic infections in lung disease: Pseudomonas infections in cystic fibrosis. Curr Opin Pharmacol. 2007;7:244-251.

33. Wesley A, Dawson K, Hewitt C, Kerr A. Clinical features of individuals with cystic fibrosis in New Zealand. N Z Med J. 1993;106:28-30.

34. Canadian Cystic Fibrosis Foundation. Canadian patient data registry national report 1998. Toronto: Canadian Cystic Fibrosis Foundation; 2001.

35. López S, Suarez M. Leucocitosis. Guías clínicas. 2006;6:1-4

36. Balke B, Schmoldt S, Häussler S, Suerbaum S, Heesemann J, Hogardt M. A German external quality survey of diagnostic microbiology of respiratory tract infections in patients with cystic fibrosis. J Cyst Fibros. 2008;7:7-14.

37. Cantón R, Molina A, Máiz L. Fibrosis quística. Monografías Neumomadrid. $2^{\mathrm{a}}$ ed. Madrid: Ergón; 2005.

38. Wat D. Impact of respiratory viral infections on cystic fibrosis. Postgrad Med J. 2003;79:201-203. 